\title{
Experimental study of bubble sweep-down in wave and current circulating tank: Part II-Bubble clouds characterization
}

\author{
Delacroix Sylvain ${ }^{1}$, Germain Grégory ${ }^{1,{ }^{*}}$, Gaurier Benoit ${ }^{1}$, Billard Jean-Yves ${ }^{2}$ \\ 1 IFREMER, Marine Structures Laboratory, 150 Quai Gambetta, 62321 Boulogne-sur-Mer, France \\ 2 IRENAV, Ecole Navale, 29240 Brest, France \\ * Corresponding author : Grégory Germain, email address : gregory.germain@ifremer.fr \\ sylvain.delacroix@ifremer.fr ; benoit.gaurier@ifremer.fr ; jyvesbilla@numericable.fr
}

\begin{abstract}
:
In this second part, images acquired from the specific trials developed in order to study the phenomenon of bubble sweep-down are analysed. A post-processing method has been developed to analyse the two air entrainment mechanisms described in the first part, for several test configurations. Bubble clouds are described in terms of depth, area and velocity for both vortex shedding and breaking wave bubble clouds. A parametric study is also performed to calculate the influence of each test parameter on the frequency of bubble generation. It is demonstrated that the occurrence of bubble clouds is proportional to the wave height, with a considerable influence of the phase shift between waves and motions. The overall results provide new elements for the understanding and the study of the phenomenon, with the final objective of obtaining a reliable tool that facilitates the design of research vessels.
\end{abstract}

\section{Highlights}

- The air entrainment mechanisms is studied by video image analysis. Depth, area and velocity bubble clouds characteristics are determined. Test parameters influence on frequency bubble generation is presented. Vortex shedding and breaking wave clouds have been characterized. Bubble clouds dependency with wave height and turbulent flow is highlighted.

Keywords : Bubble sweep-down, Experimental trials, Ship motions, Bow waves 


\section{Introduction}

Bubble generation by wave breaking and body-wave interaction is a source of diverse interest. For naval ships, this kind of bubbles generates underwater sound and a wake visible far behind the ship. In the particular case of research vessels, bubble generation must be avoided to reduce the degradation of acoustic equipment performances. Indeed, in bad weather conditions, the ship bow wave generates a significant aeration carried out by the flow under sonar locations. This phenomenon of bubble sweepdown must be prevented as much as possible in order to ensure high quality acoustic surveys Delacroix (2016), even if today there are no experimental and numerical tools allowing the exact reproduction of bubble generation by a ship's bow under 
waves and motions.

As shown in the first part of this study (Delacroix et al., 2016), bubble clouds are caused by the body-wave interaction in the bow vicinity of the ship. In the past, two kinds of wave breaking processes have been widely studied and described: spilling and plunging. In spilling breakers, turbulent fluid from the crest spills down the front face, where bubbles and droplets are formed. The plunging breaking wave is more energetic. The forward face of the crest turns into a jet that impacts the front face and the air cavity formed is entrained downward almost instantaneously into a turbulent two-phase flow. In both cases, bubble generation appears in the regions of high vorticity and turbulent breakdown. Experimental works of Duncan $(1981,1983)$ and Bonmarin (1989) described the spilling breaking waves dynamics used to develop theoretical models (Cointe and Tulin, 1994). Particle Image Velocimetry (PIV) systems enabled a better investigation of velocity and vorticity fields in quasi steady breaking waves (Lin and Rockwell, 1995; Dabiri and Gharib, 1997). Many studies (Lamarre and Melville (1994), Loewen et al. (1996), Deane and Stokes (2002), Blenkinsopp and Chaplin (2007)) have been dedicated to the measurement of void fraction and bubble size distribution in plunging breaking waves. Chanson and Cummings (1994); Cummings and Chanson (1997) studied the air entrainment mechanism by a plunging jet and developed a model to predict the sizes of the entrapped bubbles, the maximum penetration depth and the air-water gas transfer, and applied this model to plunging breaking waves. Kiger and Duncan (2012) also reviewed the mechanisms of air entrainment by a plunging jet, and the application to plunging breakers, pointing out the insufficiencies of the model to obtain a global air-entrainment model for plunging breaking waves. These works brought a lot of knowledge on the bubble generation mechanisms and are used as references in the development of numerical models of air entrainment around surface ships in stationary flows (Carrica et al., 1999; Moraga et al., 2008; Ma et al., 2011).

Despite all these studies and measurements of air-entrainment, the experimental characterization of bubble generation by the breaking bow waves of a ship is limited. The behaviour of these waves, depending on the bow geometry and the Froude number, have been well studied by (Noblesse et al., 2008, 2013) and Delhommeau et al. (2009). However, studies of bubble generation in this configuration are scarce. Waniewski et al. (2001) used IVFM (Impedance Void Fraction Meter) to measure the void fraction in a breaking bow wave simulated by a deflecting plate. Shakeri et al. (2009) and Tavakolinejad (2010) have developed a 2D $+\mathrm{t}$ technique allowing the simulation of a $21.03 \mathrm{~m}$ model at 27.5 knots during which bubble sizes and void fraction have been acquired by a shadowgraph measurement system. Both studies focused on thin and fast ship's steady bow wave in calm seas. The influence of sea states and motions, which are significant on the acoustic survey efficiency, is not considered.

In this paper, we study bubble generation around a ship model submitted to waves and motions in order to understand the mechanisms of air entrainment at real scale despite the similarity issues discussed in the first part of this study. A quantification of these mechanisms is performed on a 1/30 model of the Pourquoi pas?, corresponding to a ship model of $3.13 \mathrm{~m}$ length between perpendiculars (Lpp), $0.67 \mathrm{~m}$ beam and $0.18 \mathrm{~m}$ draft, in a wave and current tank. Delacroix et al. (2016) describes the experimental set-up developed to reproduce the real conditions of bubble sweep-down in a circulating tank (presented figure 1). Acquisition systems 
and first observations of bubble generation are also detailed. This study was facilitated by the use of a hexapod allowing to consider the four base configurations: 1/ with current only, 2/ with current and waves, $3 /$ with current and motions and 4/ with current, waves and motions. Two phenomena of air entrainment have been observed: air entrainment by vortex shedding or by the breaking bow waves, for which a schematic description is given in figure 2 . The distinct bubble clouds (as opposed to single bubbles) frequency are recalled on figure 3.

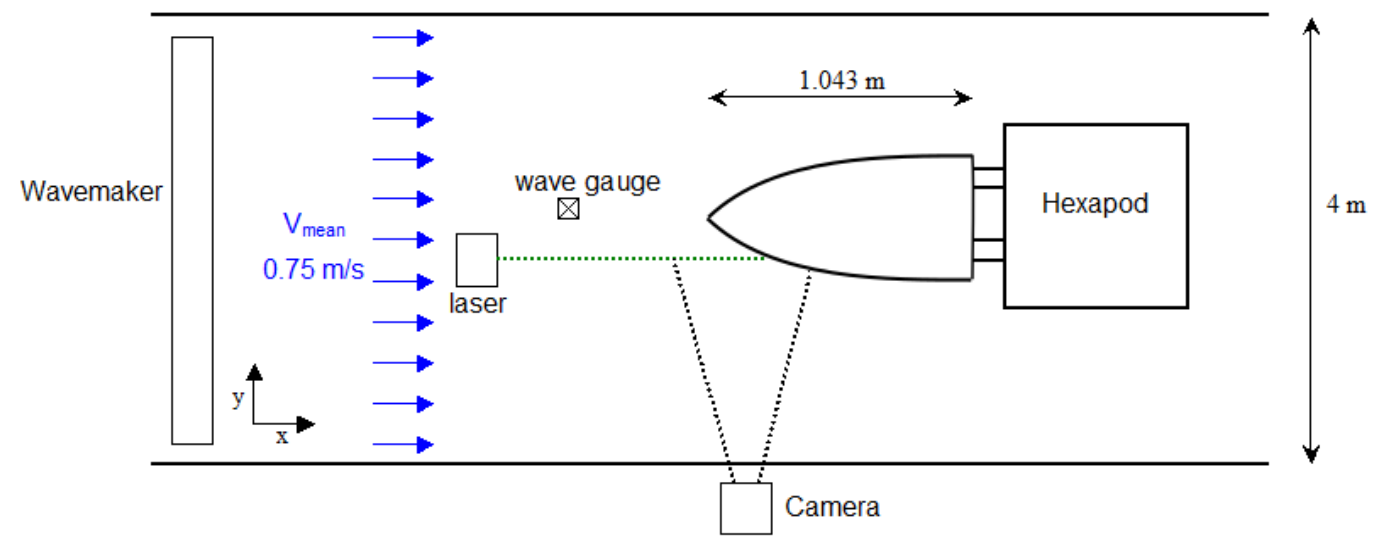

Figure 1: Drawing of the experimental set-up showing the wavemaker and the light sheet generation system location
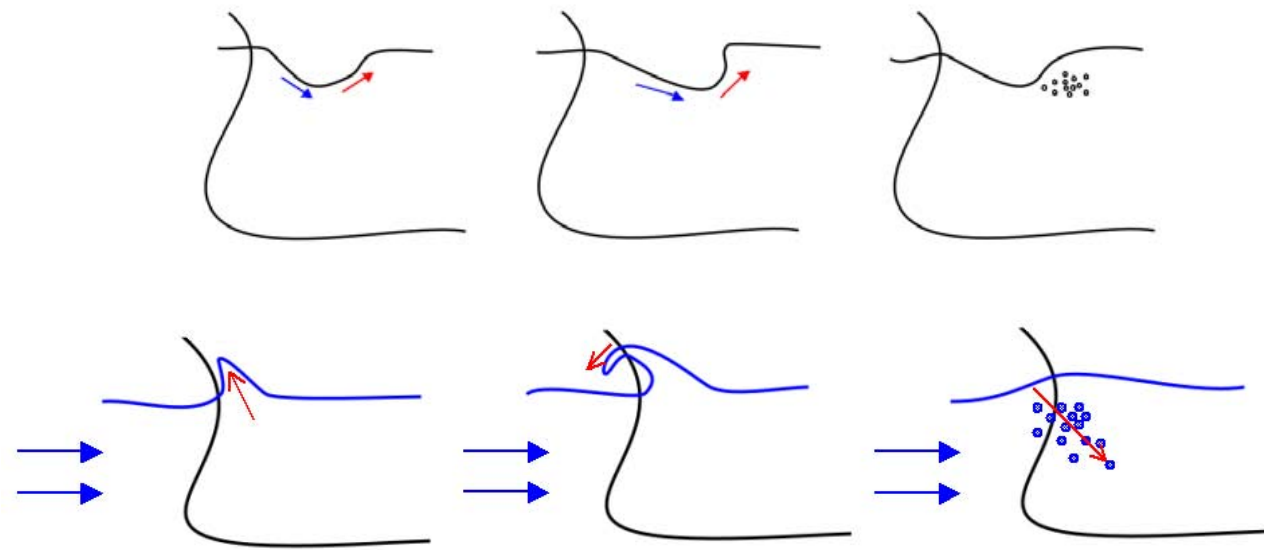

Figure 2: Schematic description of the vortex shedding and breaking wave clouds.

The interaction between the turbulent incoming flow and the bow generates low frequent vortex shedding clouds (configuration 1). This phenomenon is present in the four configurations with similar frequencies and the hull motions may amplify the amount of air entrapped. Configuration 2 shows that the impact of waves on the bow generates breaking waves and a higher frequency of bubble generation. Configuration 3 with motion also generates some breaking waves. The fourth configuration with waves and motions corresponds to the highest frequency of bubble clouds. 


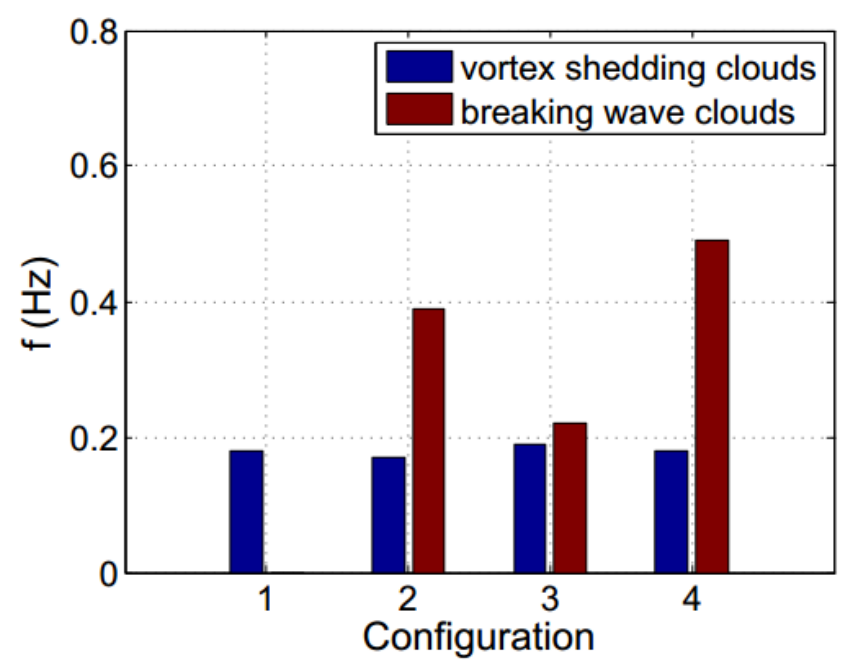

Figure 3: Frequency of occurrence of the vortex shedding and breaking wave clouds for the 4 base configurations.

In this second part, the methodology of image post-processing is presented in section 2, while the results in terms of bubble clouds characterization in section 3. Section 4 is devoted to study the influence of several parameters on bubble generation, and finally a discussion about the results and their generalization are discussed in section 5 .

\section{Configurations and analysis method}

The experimental setup was developed to simulate real conditions of bubble sweep-down in a wave and current circulating tank. Many parameters are involved in the bubble generation (current speed, waves and motion characteristics...). Several configurations have been carried out to characterize the phenomenon in the tank and are detailed in this section. Typical bubble clouds are studied to describe the mechanisms of air entrainment. The image analysis method to obtain the bubble clouds characteristics and the clouds occurrence in each configuration is described below.

\section{$2.1 \quad$ Test Configurations}

Numerous tests were conducted to characterize the influence of each parameter of the experiment on the phenomenon. The parameters of these tests are given in table 1.

- Runs 1-4 for the 4 base configurations, allowing the independent study of waves and motions;

- Runs 5-12 to study the influence of the wave amplitude and frequency. The amplitude A entered in table 1 referred to the wavemaker input and is not exactly the mean amplitude measured. $A=20,40,55,65$ and $85 \mathrm{~mm}$ with $f=0.85 \mathrm{~Hz}$ respectively for runs 5 to 9 and $f=0.65,0.75$ and $1.00 \mathrm{~Hz}$ with $A=75 \mathrm{~mm}$ for runs 10 to 12 ; 
- Runs 13-18 to study the influence of motions amplitude and frequency. The Amplitude Coefficient $A C=0.75,1.25$ and 1.50 with $f=0.85 \mathrm{~Hz}$ for runs 13 to 15 and $f=$ $0.65,0.75$ and $1.00 \mathrm{~Hz}$ with $A C=1$ for runs 16 to 18 ;

- Runs 19-26 to study the influence of current speed: the four base configurations were repeated for the equivalent speeds of 4 and 6 knots;

- Runs 27-30 to study the influence of the synchronism between the waves and motions, configuration 4 was repeated with four different phase shifts;

- Runs 31 and 32 to study the influence of the turbulence of the flow on bubble generation in the circulating tank. The presence of the wavemaker upstream generates a highly turbulent flow with a Turbulent Intensity $T I=15 \%$. In order to work with similar incoming flows, configurations 1 and 3, without waves, were also performed with the wavemaker upstream. These two configurations were repeated without the wavemaker, which corresponds to a turbulence reduction down to $T I=3 \%$.

Table 1: Description of the four base configurations used to study bubble sweep-down. The data in brackets refer to the variation of each parameter for the parametric study tests.

\begin{tabular}{l|l|ll|ll}
\hline Config. & $\begin{array}{l}\text { V } \\
(\text { knots })\end{array}$ & $\begin{array}{l}\text { Waves }(\mathrm{mm}) \\
\mathrm{A}(\mathrm{Hz})\end{array}$ & $\begin{array}{l}\text { Motions } \\
\text { AC }\end{array}$ & $\mathrm{f}(\mathrm{Hz})$ \\
\hline 1 & 8 & No & No & No & No \\
$(19-20)$ & $(4-6)$ & - & - & - & - \\
\hline 2 & 8 & 75 & 0.85 & No & No \\
$(5-9)$ & - & $(20-85)$ & - & - & - \\
$(10-12)$ & - & - & $(0.65-1.0)$ & - & - \\
$(21-22)$ & $(4-6)$ & - & - & - & - \\
\hline 3 & 8 & No & No & 1 & 0.85 \\
$(13-15)$ & - & - & - & $(0.75-1.5)$ & - \\
$(16-18)$ & - & - & - & - & $(0.65-1.0)$ \\
$(23-24)$ & $(4-6)$ & - & - & - & - \\
\hline 4 & 8 & 75 & 0.85 & 1 & 0.85 \\
$(25-26)$ & $(4-6)$ & - & - & - & - \\
\hline
\end{tabular}

The acquisition system simultaneously records images, waves and motions measurements. The images were acquired at a $15 \mathrm{~Hz}$ frequency on periods of 180 seconds for each run, corresponding to 2700 images.

\subsection{Image Analysis Method}

The image analysis allows the characterization of the phenomenon of bubble generation in a circulating tank and a better understanding of the influence of each parameter of the experiment.

On figure 4, several steps of the image analysis method are shown. All the images are exported from the acquisition system and post-processing software Dynamic Studio (Dantec, 
2010), and treated via a grey level analysis. It should be noted here that the $2 \mathrm{D}$ detection of a 3D phenomenon induces a measurement bias which should be taken into account in future works.

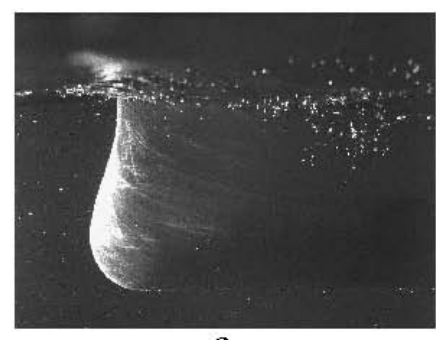

a

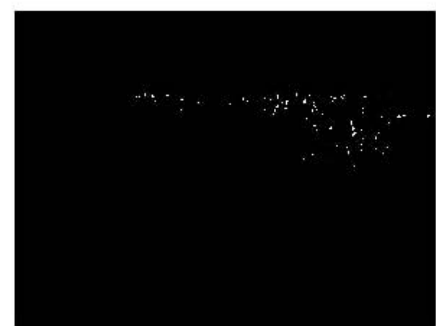

d

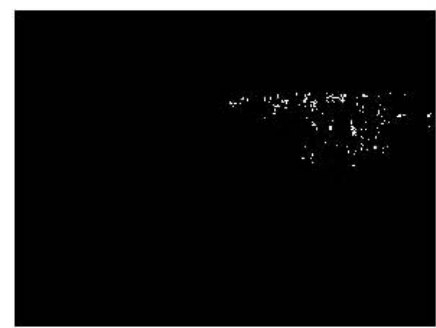

g

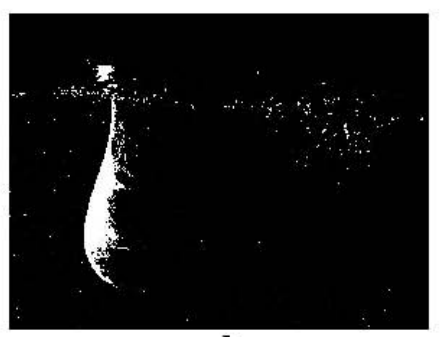

b

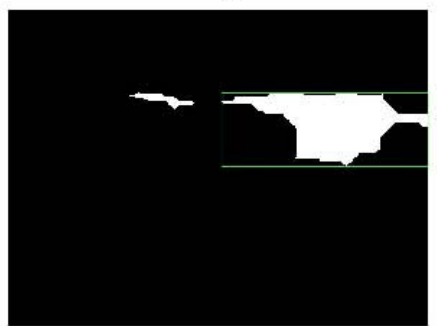

e

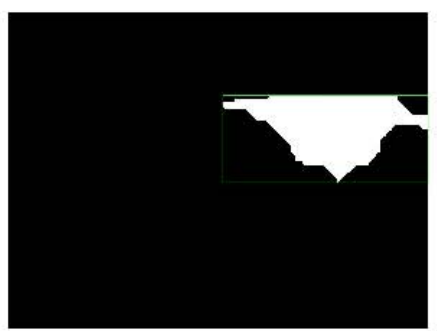

h

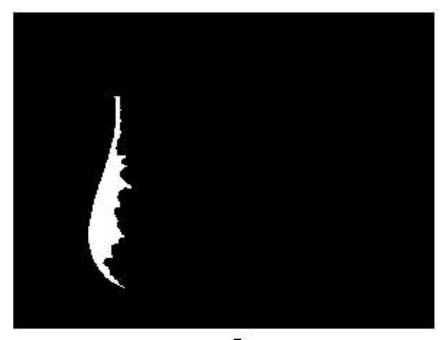

c

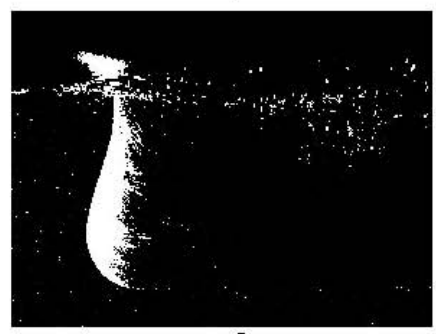

$\mathbf{f}$

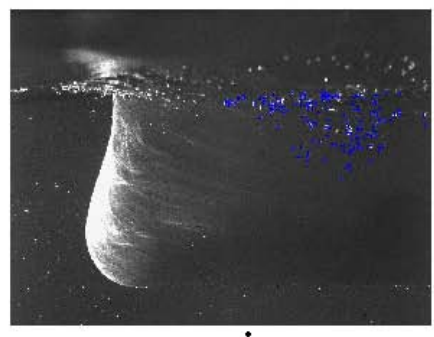

i

Figure 4: Visualization of several steps of the image analysis, from the raw image (a) to the aeration detection $(\mathrm{g})$ and the cloud area location $(\mathrm{h})$.

The raw image (a) is converted to a binary image (b) for edge detection, based on the Prewitt method (Prewitt, 1970), with a first grey level. The presence of hull reflections is clearly indicated and this image allows to detect the bow area (c) corresponding to the largest zone detected. The air-water interface at the bow is then easily detectable at the top of this area. In this paper the free surface is assumed to be horizontal on the image. A specific study is undertaken to obtain a better detection of the free surface (Dussol, 2015) but this approximation does not prevent the study of bubble clouds properties. To isolate the bubble light reflections from other reflections (ship bow, free surface,...), we focus on a specific area of the image: below the free surface line and downstream of the bow area detection. We also limited the automatic detection of bubbles with equivalent diameters (defined as $d=\sqrt{4 \times A r e a / \pi}$ ) larger than $d_{\min }=0.6 \mathrm{~mm}$ to avoid any confusions between the bubbles and other tank particles. The bubbles are then identified (d), as the bubble clouds (e) defined as the area encompassing sufficiently close to the detected bubbles.

A second loop is performed with a higher grey level (f) to obtain more detailed information in the location of the largest cloud. Image $(\mathrm{g})$ shows the final aerations detected in the region. The majority of these detections are single bubbles but may in some cases be an agglomeration 
of several bubbles. Hereafter it is referred to as "aerations". Image (h) shows the final cloud area detection and on image (i) the aerations detected are marked on the raw image.
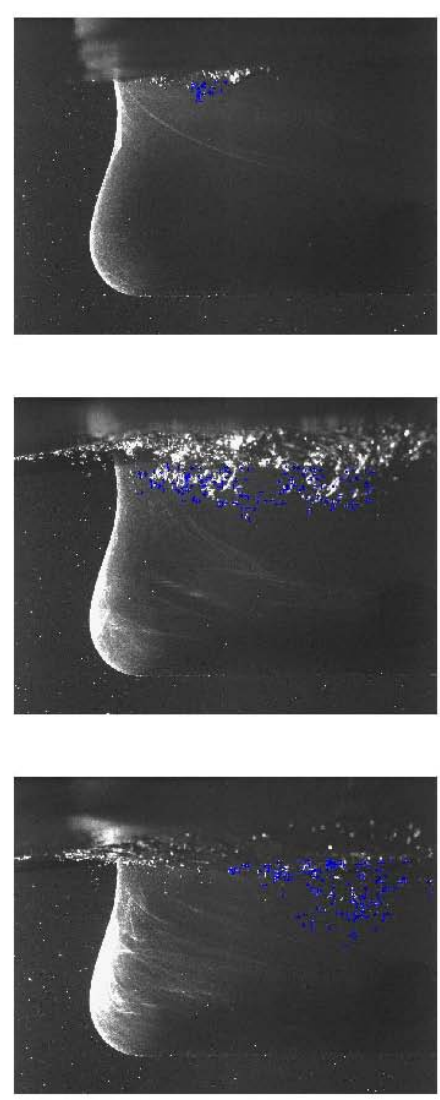
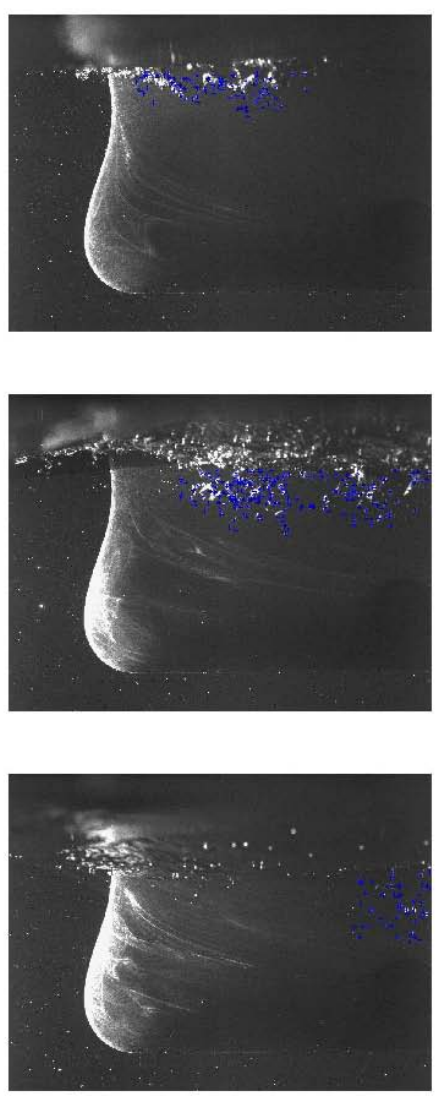
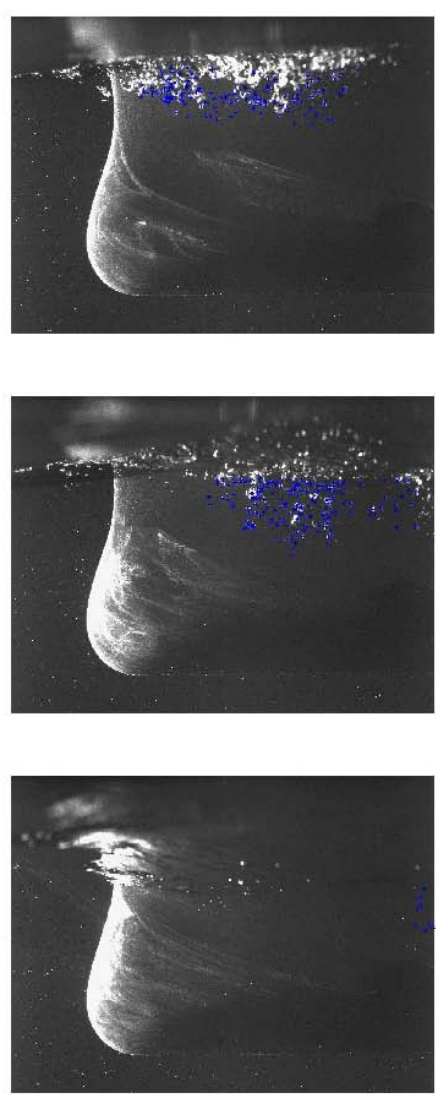

Figure 5: Sequence of a breaking wave bubble cloud, from configuration 2 with current and waves, with the aerations detected by the image analysis marked in blue.

The application of the image analysis on a breaking wave cloud sequence is presented in figures 5 and 6 . Figure 5 shows the detection of aerations on each image while figure 6 shows the evolution of the bubble clouds area. This image analysis method allows an automatic detection of bubbles. In this way the bubble cloud frequency, but also the cloud area (as shown in figure 6), the aeration size and quantity, the cloud density (defined as the ratio of aerations area on the cloud area), the maximal depth of penetration and the global cloud evolution (horizontal and vertical velocities) can be calculated. The instrumentation system calibration led to a space discretization of $0.225 \mathrm{~mm}$.

Due to the complexity of the experiments, the light is not always perfect and may vary because of the wave and model motions. Moreover, these analyses do not take into account depth of field, which affects the precision of the measurements. However, this post-processing provides many valuable data for the characterization of the bubble generation. The relatively limited number of images enables a visual verification of the aerations detected on each image of the sequence. It also enables to fix the parameters used during the process, like the grey levels or the definition of the cloud, with very low potential errors on the calculated properties (bubble cloud frequency, area, depth, ...), as illustrated figures 7 to 9 . The error analysis on 

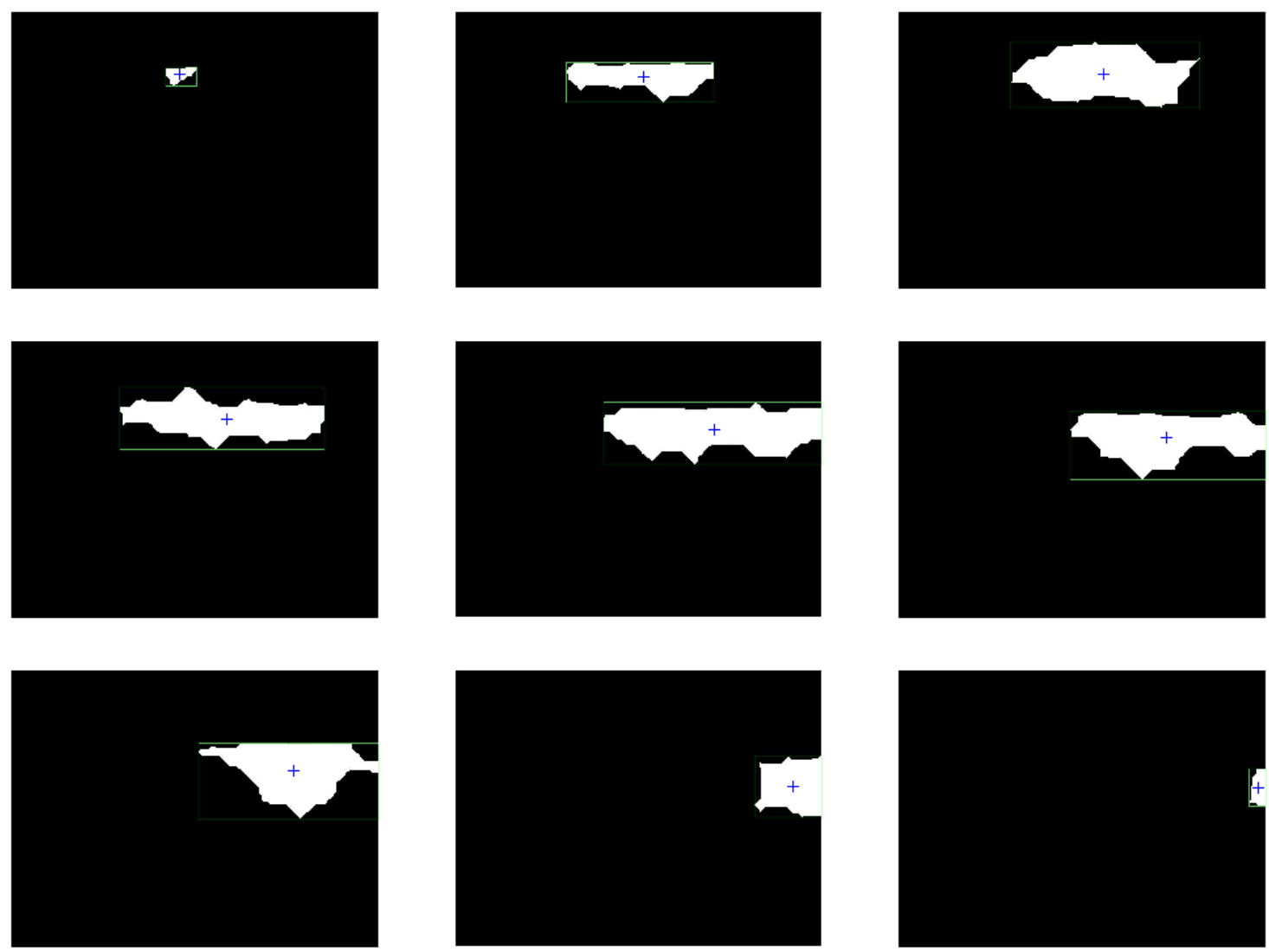

Figure 6: Evolution of the bubble cloud area (sequence of the breaking wave showed on figure $5)$. The ' + ' gives the location of the bubbles cloud center.

these properties calculations was detailed in Delacroix (2015).
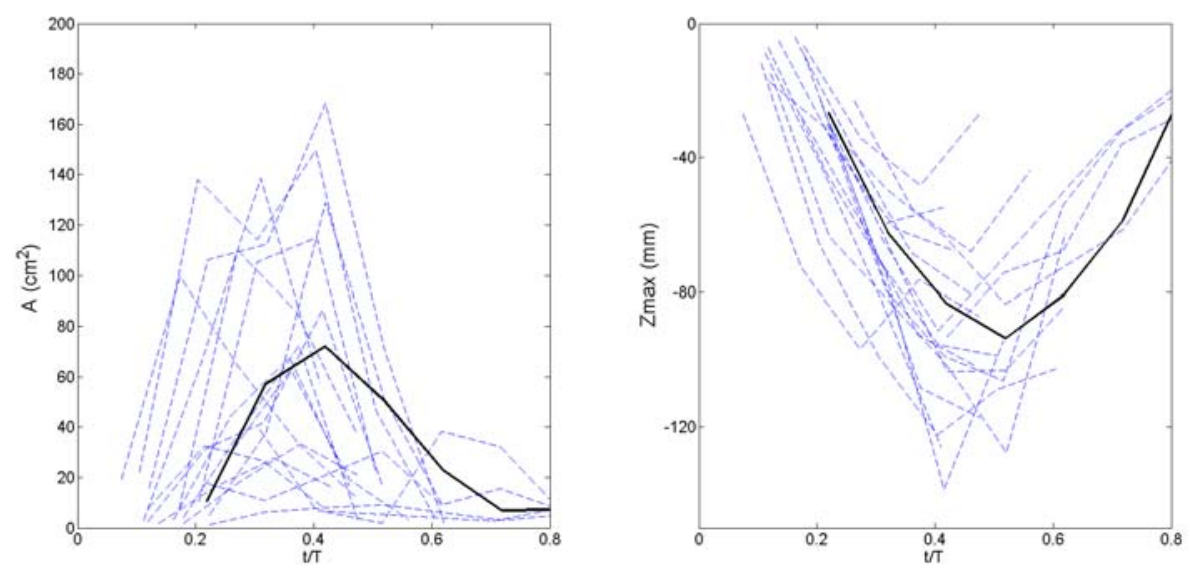

Figure 7: Bubble clouds properties (Area and maximal depth) in configuration 2: current and waves, obtained by the image analysis method.

Figure 7 shows the evolution of the cloud areas (left graph) and the maximum bubble depths (right graph) for the second configuration. As explained in detail in the following section, each dotted line represents a significant cloud of bubbles. The bold black curves are the average of 
these properties, obtained by taking into account only the deepest clouds (exceeding $80 \mathrm{~mm}$ of maximum depth).

The influence of the first grey-level threshold choice is shown on figure 8. We can notice that this threshold level has very little influence on the maximum depth detected. However, the areas obtained vary more significantly.
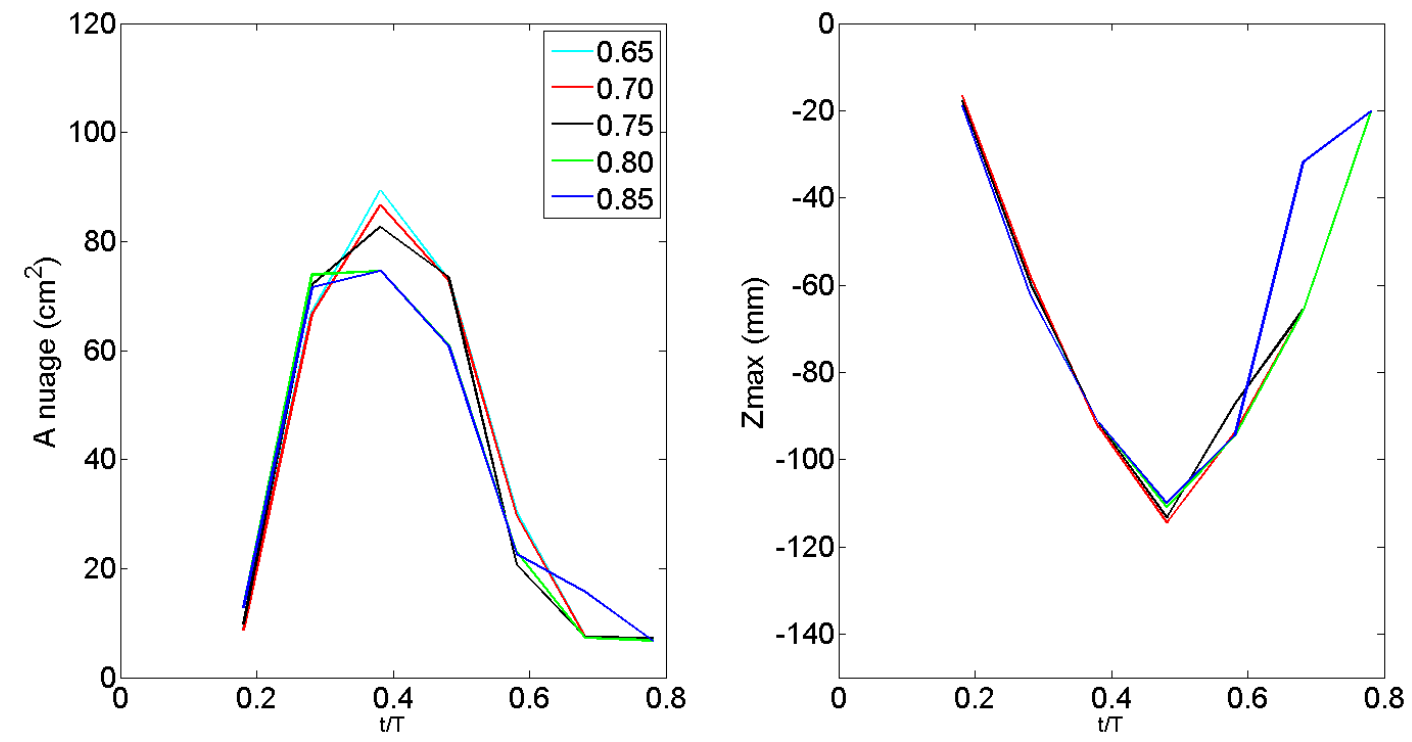

Figure 8: Influence of the first grey level threshold on the two main mean properties: clouds area and maximum depth

The average described on these graphs are obtained for 5 thresholds of grey levels: $0.65 ; 0.70$; $0.75 ; 0.80$ and 0.85 . The grey levels 0 and 1 correspond to a completely black or white pixel respectively. If the grey level threshold is low it is easier to detect reflections, even less intense, but if the grey threshold is high only the most intense reflections are detected, as illustrated in figure 9. On the image on the left, as the threshold is too low (0.55) reflections that are not caused by bubbles are detected. The right hand side image represents a too high threshold, many bubbles not being detected.

The first step in the selection of a grey level threshold is to verify that neither situation is occurring in the entire sequence. Given the number of images per sequence (1530) this step may be done by operator visualisation. On each sequence we can verify that the detection criteria are adequately chosen.

The curves in figure 8 give an idea of the measurements accuracy. The thresholds of 0.85 and 0.80 are too high and are not taken into account. Errors of about $5 \mathrm{~cm}^{2}$ are observed for the mean clouds area (6.2\% of error) and of a few millimetres for the maximum depth (an error of $3.6 \%$ ). This method is used in the next section for the characterization of the dynamics of bubble clouds.

\section{Characterization of bubble clouds}

Bubble clouds of the four base configurations are analysed thanks to the image post-processing described in section 2.2. Figures 10 to 13 show the evolution of the significant clouds, defined 

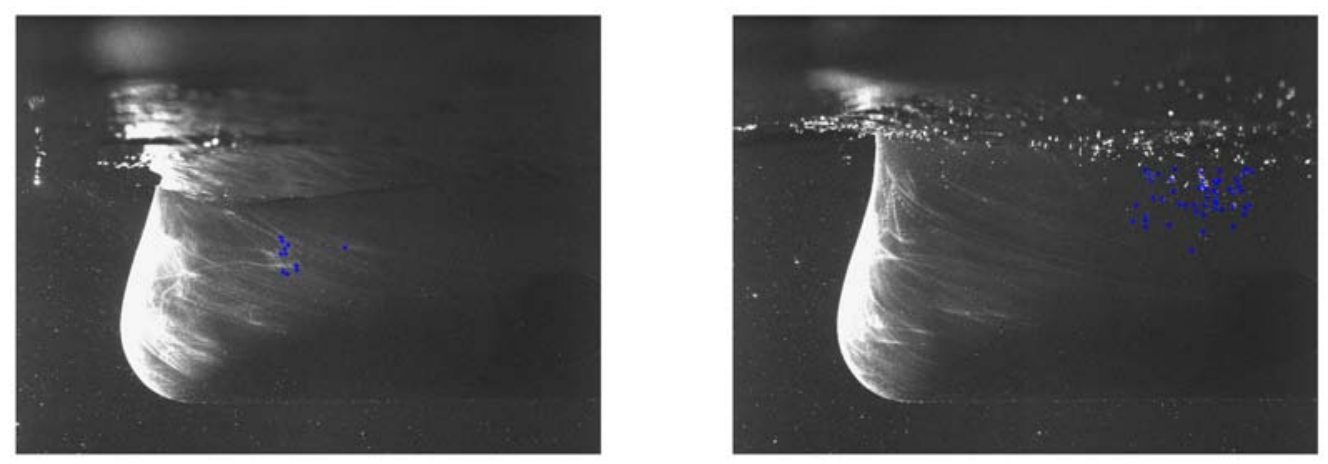

Figure 9: Visual setting of the grey level threshold. Left: threshold is too low $(0,55)$. Right: threshold is too high $(0,85)$.

as being observable on at least four consecutive images. Each blue dotted curve corresponds to a significant cloud, while the black bold line corresponds to the mean value. Three properties are represented on these figures: the cloud area, the maximal depth and the vertical velocity of the clouds (determined from the cloud center locations). All the figures abscissa correspond to the dimensionless time $t / T$ with $T$ the wave or motion period $T=1 / 0.85 \approx 1.18 \mathrm{~s}$.

\subsection{Configuration 1: Current only}

In the first configuration (figure 10), the instant $t=0$ corresponds to the first aeration detection for each cloud. All the clouds are caused by air entrainment by vortex shedding. The area of the clouds is always lower than $0.5 \cdot 10^{4} \mathrm{~mm}^{2}$. The maximal penetration depth of the bubbles is slightly above $50 \mathrm{~mm}$. The vertical velocity of the clouds is on average close to zero during the first half of the cloud life and tends to be positive afterwards, the bubbles rising to the surface.
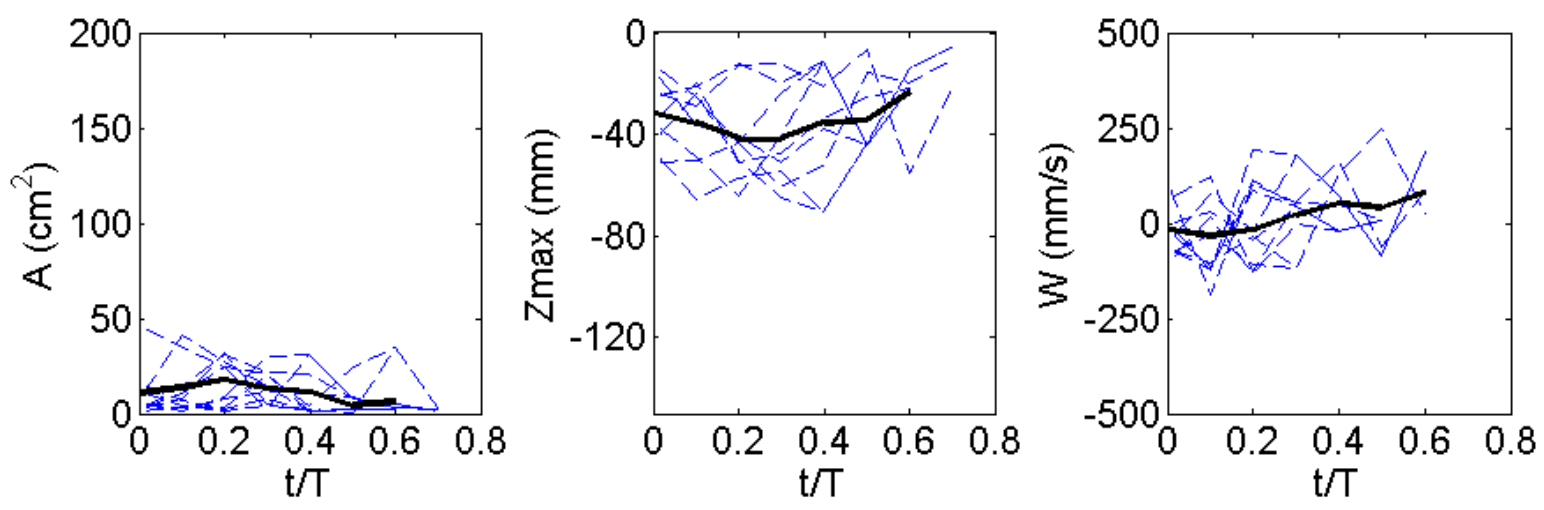

Figure 10: Bubble clouds properties (Area, maximal depth and vertical velocity) in configuration 1: current only.

\subsection{Configuration 2: Waves and current}

In the second configuration (figure 11), the instant $t=0$ corresponds to the crest of the incoming wave at the bow. During this test the great majority of significant clouds are due to breaking waves. In order to characterize this phenomenon, only the breaking wave clouds are selected to 
calculate the mean value. The inception of bubble clouds visualization happens between 0.1 and $0.3 \mathrm{~T}$ after the crest of the wave. The cloud properties are very different from the vortex shedding ones. The cloud areas and depths present a clear peak between 0.3 and $0.5 \mathrm{~T}$. The maximal cloud area is often 3 times higher than that of the vortex shedding one (up to $1.8 \cdot 10^{4} \mathrm{~mm}^{2}$ ). The maximal depth penetration reaches $140 \mathrm{~mm}$. Even if this value is significantly higher than the vortex shedding cloud depth, the breaking wave clouds never pass under the hull (of $182 \mathrm{~mm}$ draft). Finally the vertical cloud velocities are close to $-0.25 \mathrm{~m} / \mathrm{s}$ after the breaking events, tend to zero between 0.4 and $0.5 \mathrm{~T}$ and increase to a rise velocity of $0.25 \mathrm{~m} / \mathrm{s}$ between 0.5 and $0.7 T$.

These values can be compared to the velocities generated by the waves. Applying the theory of Airy, the orbital velocity in infinite depth approximation is given by:

$$
w=A \omega e^{k z} \sin (k x-\omega t)
$$

with $\mathrm{A}$ the amplitude, $\omega$ the temporal angular frequency and $\mathrm{k}$ the wave number. Thus, the maximum value of the vertical orbital velocity near the free surface is $w=A \omega=0.18 \mathrm{~m} / \mathrm{s}$ for a $33 \mathrm{~mm}$ amplitude regular wave. In this case, the interaction of the incoming wave with the bow increases the vertical component by a factor 1.4, showing the increase of amount of energy at the start-up of clouds formation.
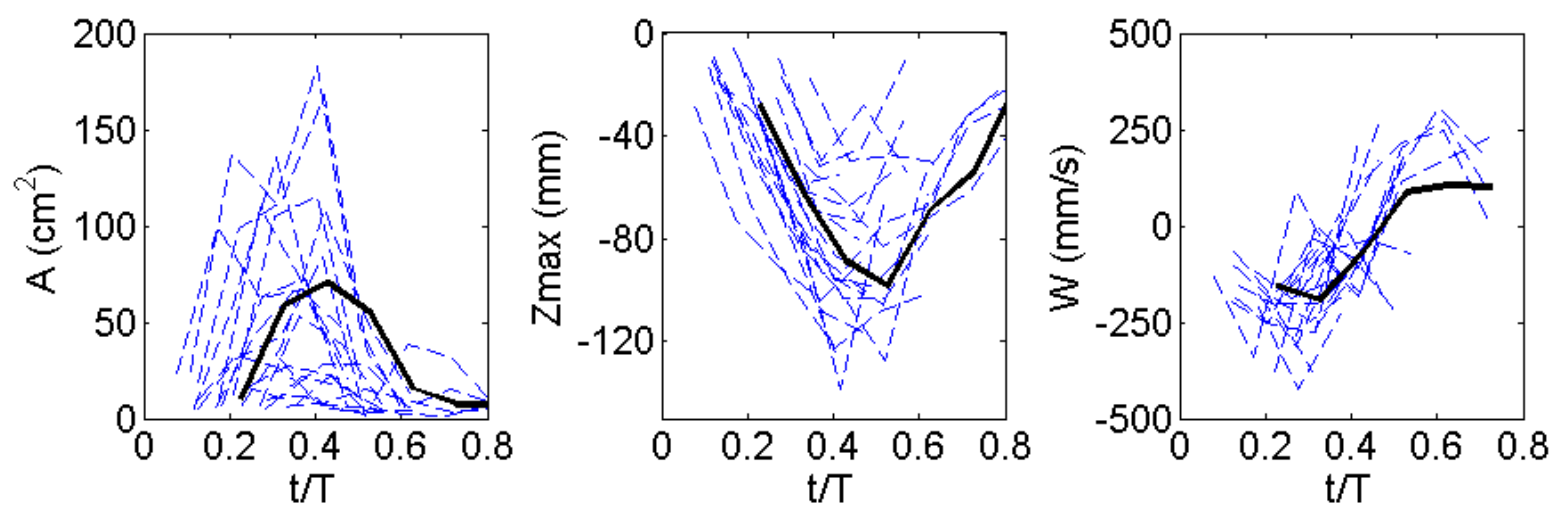

Figure 11: Bubble clouds properties (Area, maximal depth and vertical velocity) in configuration 2: current and waves.

\subsection{Configuration 3: Motions and current}

In the third configuration (figure 12), the instant $t=0$ corresponds to the peak of the pitch (highest position of the bow). In this case, even if the frequency of breaking wave cloud is as important as the vortex shedding cloud frequency, these events are not as energetic as in the configuration with the waves impacting the bow. The significant clouds with current and motions are mostly due to vortex shedding clouds with model movements favouring the air entrainment, as confirmed by the cloud properties close to the first configuration. The variation of the vertical velocities are higher than in the first configuration, probably because of the amplification of the vortex by the model's motions. The maximal cloud area and depth penetration are respectively 
$0.8 \cdot 10^{4} \mathrm{~mm}^{2}$ and $84 \mathrm{~mm}$. The vertical velocities vary between -0.3 and $0.46 \mathrm{~m} / \mathrm{s}$.
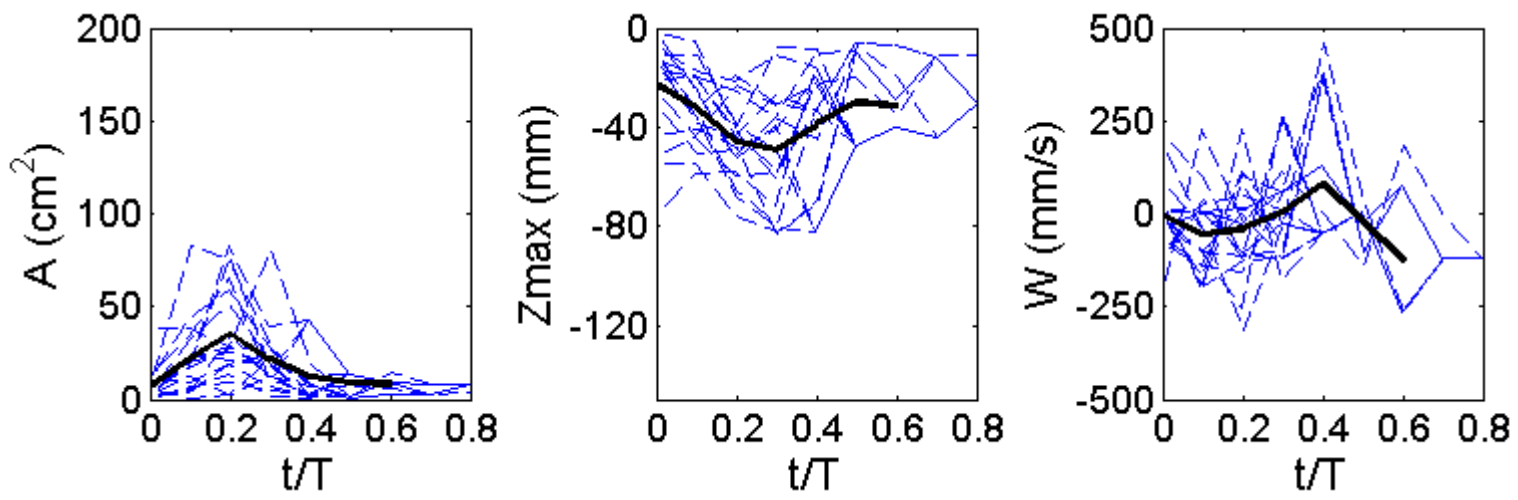

Figure 12: Bubble cloud properties (Area, maximal depth and vertical velocity) in configuration 3: current and ship motions.

\subsection{Configuration 4: Waves and Motions}

In the fourth configuration (figure 13), the instant $t=0$ corresponds to the crest of the incoming wave at the bow, as in configuration 2. The number of significant clouds is the highest in this case. The properties of the clouds are similar to those in the second configuration, attesting the predominance of breaking wave clouds. The maximal cloud area and depth penetration are also very close (respectively $1.4 \cdot 10^{4} \mathrm{~mm}^{2}$ and $146 \mathrm{~mm}$ ), while the absolute values of the vertical velocities can be higher (from -0.5 up to $0.5 \mathrm{~mm} / \mathrm{s}$ ).
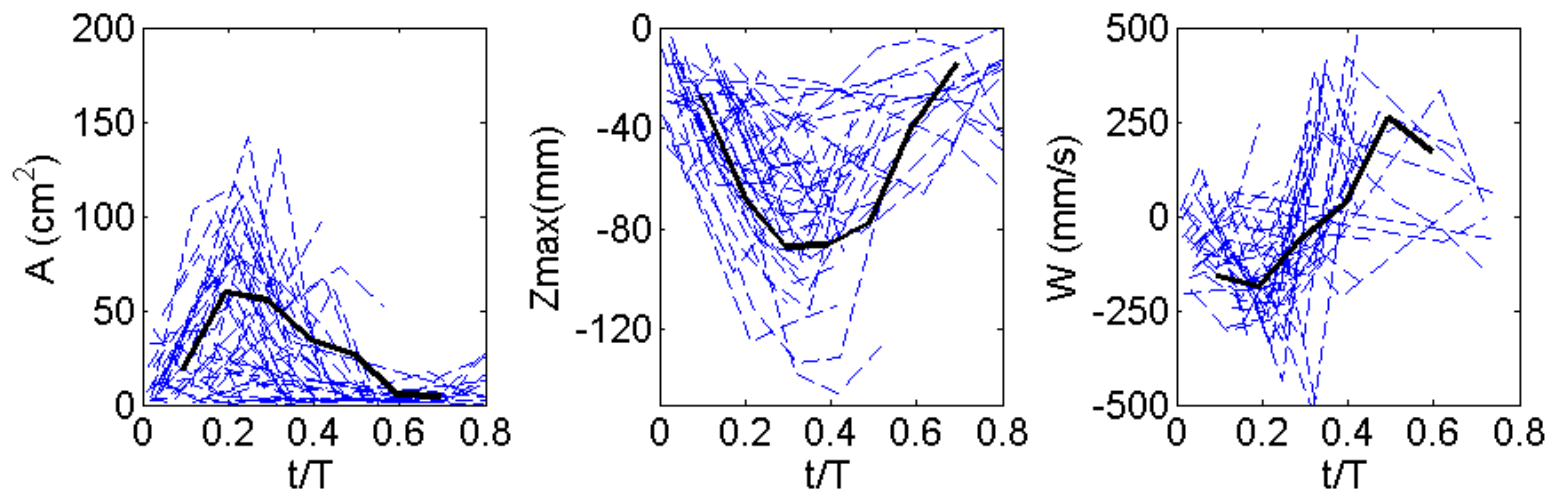

Figure 13: Bubble cloud properties (Area, maximal depth and vertical velocity) in configuration 4: current, waves and motions.

The mean values for the four configurations are compared on figure 14. On these graphs the properties are made dimensionless. The area of the cloud is divided by that of the rectangle defined by the draft $D=0.182 \mathrm{~m}$ and the length $l=0.2 \cdot L_{p p}=0.626 \mathrm{~m}: a=A /(D \cdot l)$. The maximal depth is divided by the draft, $z=Z_{\max } / D$ and the vertical velocity is divided by the incoming flow mean velocity, $w=W / U$. This analysis confirms the description of air entrainment mechanisms posited from direct visualisation in the first part of the article. Vortex shedding clouds (configuration 1) are relatively small and stay close to the surface, while breaking wave clouds (configurations 2 and 4) may be larger and entrained deeper a few instants after 
the crest of the incoming wave. The main difference between configurations 2 and 4 is that the breaking event is generated sooner in the wave period in the fourth configuration, by the impact between the wave and the bow motion.
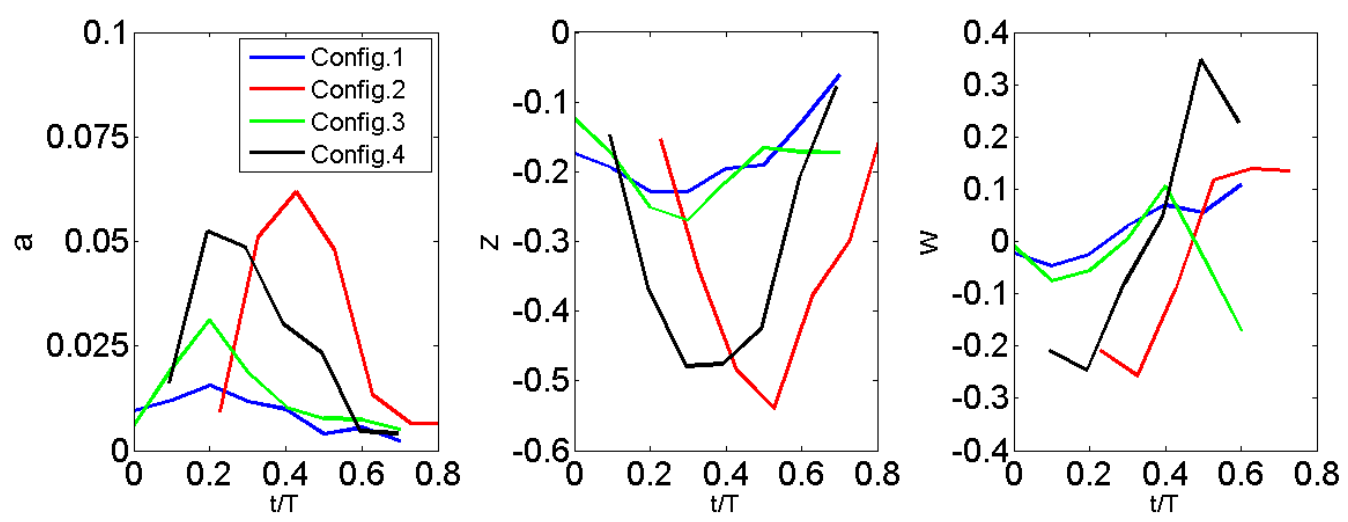

Figure 14: Comparison of the dimensionless bubble cloud properties for the four configurations.

\section{Parametric study of the occurrence of bubble clouds}

Section 3 has allowed to characterize the two kinds of bubble clouds generated in the circulating tank. The influence of the interaction of an incoming turbulent flow on the bow of the Pourquoi pas?, of the waves and finally the motions, are obtained through the four base configurations. In this section, the various parameters of these tests are modified in order to understand the influence of each of them on the bubble clouds occurrence.

\subsection{Influence of the waves characteristics}

As explained in the first section, the impact of the waves on the bow generates breaking wave clouds, which are most likely to be entrained deeply and disturb acoustic signals. Waves are consequently the most important parameter. However, they are also difficult to control because of the interaction of the current and the wavemaker. The fluctuations of the wave amplitudes are significant (figure 15).

The wave signal from the second configuration with waves has been analysed to sort the waves according to their height. Figure 16 shows the evolution of the clouds' depth for three wave height levels. The graph on the left corresponds to the significant bubble clouds observed for the highest third of waves $(45[\mathrm{~mm}]<A)$. In the center and on the right stand the equivalent graphs for the intermediate-amplitude third $(35<A<45)$ and the lower-amplitude third $(A<35)$, respectively. As we can see, the bubble generation is directly related to the wave amplitude, with a greatest number of bubble clouds on the left-most graph. This analysis also shows that the influence of the wave amplitude on bubble clouds frequency should not be studied directly from the mean clouds frequency of several amplitude input tests alone.

For this purpose all the tests with current and waves (configurations 2 and 5 through 9) have been analysed as an independent database. All waves signals have been divided by groups of wave height, every $10 \mathrm{~mm}$. The bubble clouds frequency is calculated for each group as can 


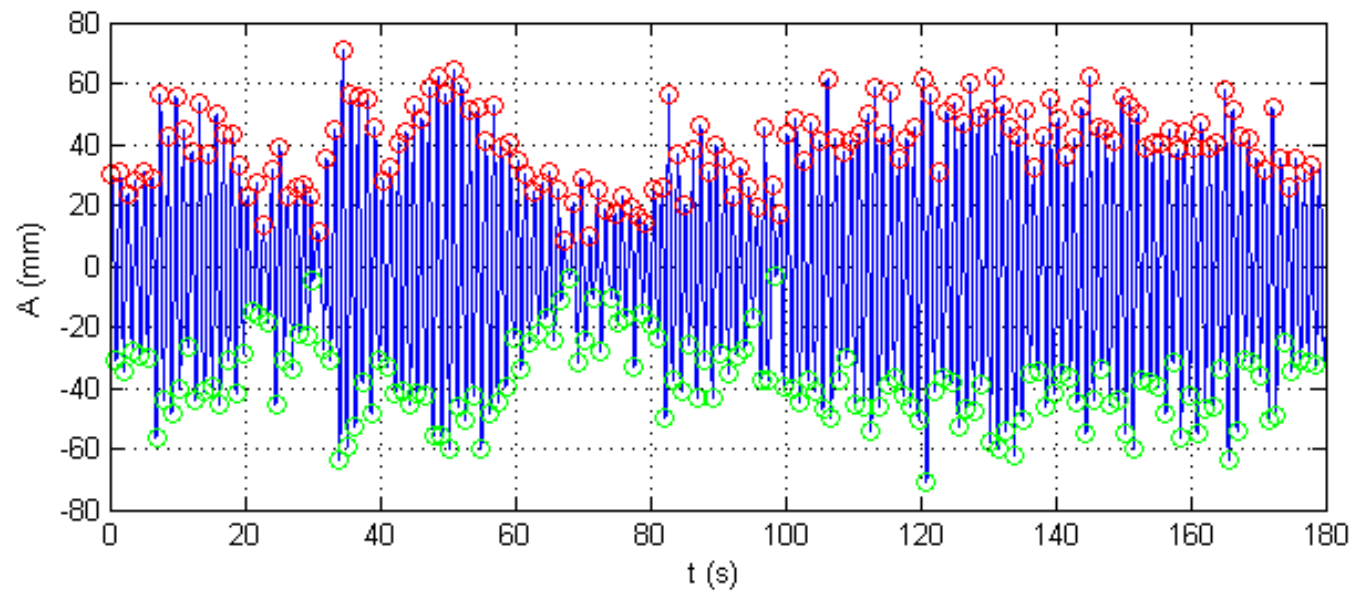

Figure 15: Fluctuation of the wave amplitude. Signal recorded by the wave gauge $1 \mathrm{~m}$ upstream from the bow
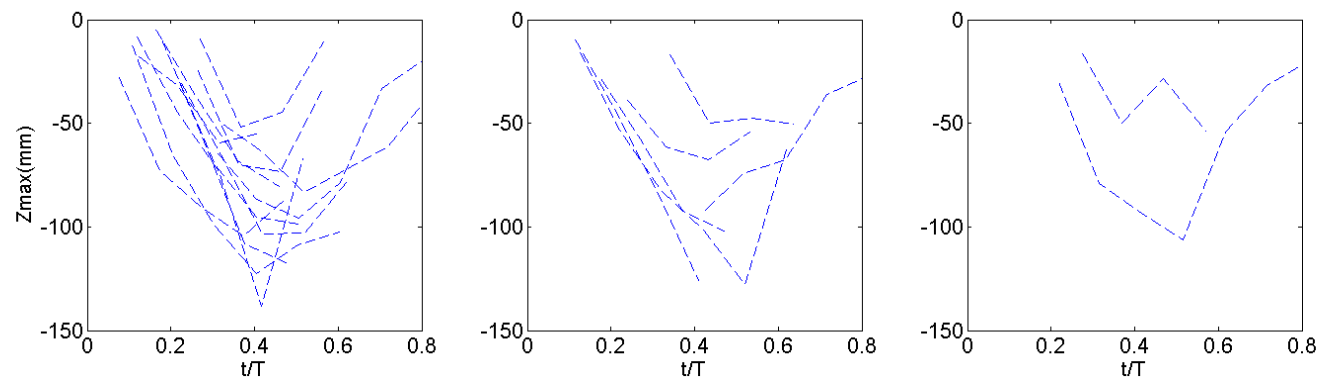

Figure 16: Maximal depth evolution of the significant clouds of the second configuration. Left: for the third of the highest wave amplitude. Center: for the third of intermediate wave amplitude. Right: for the third of the lowest wave amplitude.

be seen on figure 17 (left). This result confirms that the clouds frequency in the configuration with waves and current increases linearly with increasing wave height, with a coefficient of determination $R^{2}=0.91$.

Graph 17 (right) corresponds to the analysis of configurations 2 and 10 to 12 with wave frequencies of respectively $0.85,0.65,0.75$ and $1.00 \mathrm{~Hz}$. For all these tests, only the bubble clouds generated by waves corresponding to the desired test conditions (wave height $=66 \mathrm{~mm}$ plus or minus $5 \mathrm{~mm}$ ) were considered. The cloud frequency reaches a maximum between wave frequencies of 0.75 and $0.85 \mathrm{~Hz}$ and decreases until $0.3 \mathrm{~Hz}$ for a wave frequency of $1.00 \mathrm{~Hz}$. For such frequency, the wavelength becomes too short, the impact on the bow is smoother and the amount of breaking event is reduced.

\subsection{Influence of ship dynamics}

Another main parameter is the current's velocity. The four base configurations have been repeated for currents corresponding to 4,6 and 8 knots (respectively $0.38,0.56$ and $0.75 \mathrm{~m} / \mathrm{s}$ in the tank at a $1 / 30$ scale). As we can see on figure 18, the bubble cloud frequency is notably lower at $6 \operatorname{knots}(f=0.22 \mathrm{~Hz}$ with waves and motions) and minimal at $4 \operatorname{knots}(f=0.06 \mathrm{~Hz})$. For these velocities the waves do not generate more bubble clouds than the motions. 

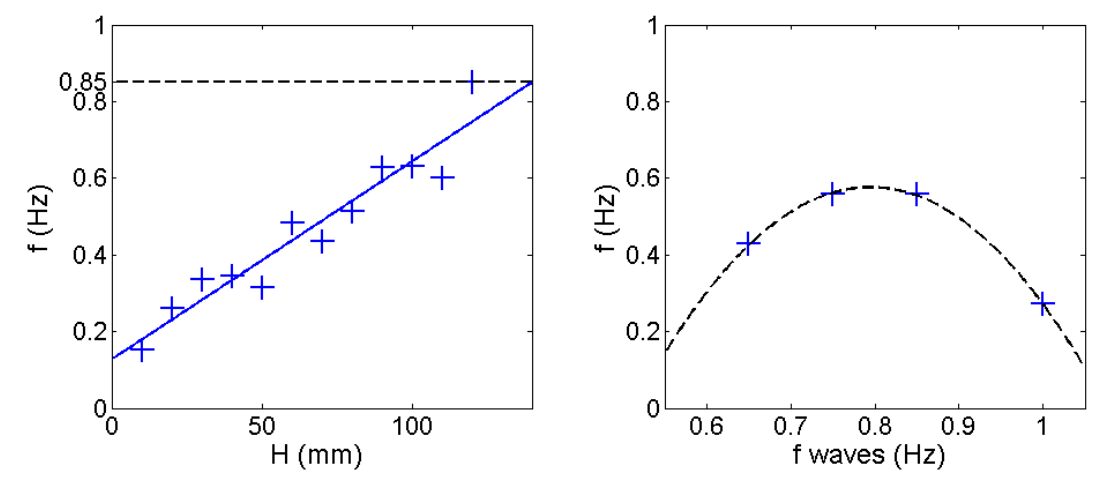

Figure 17: Influence of the wave parameters on the bubble cloud frequency. Left: cloud frequency against wave height, and linear regression $\left(R^{2}=0.91\right)$. Right: cloud frequency against wave frequency, and quadratic regression $\left(R^{2}=0.99\right)$.

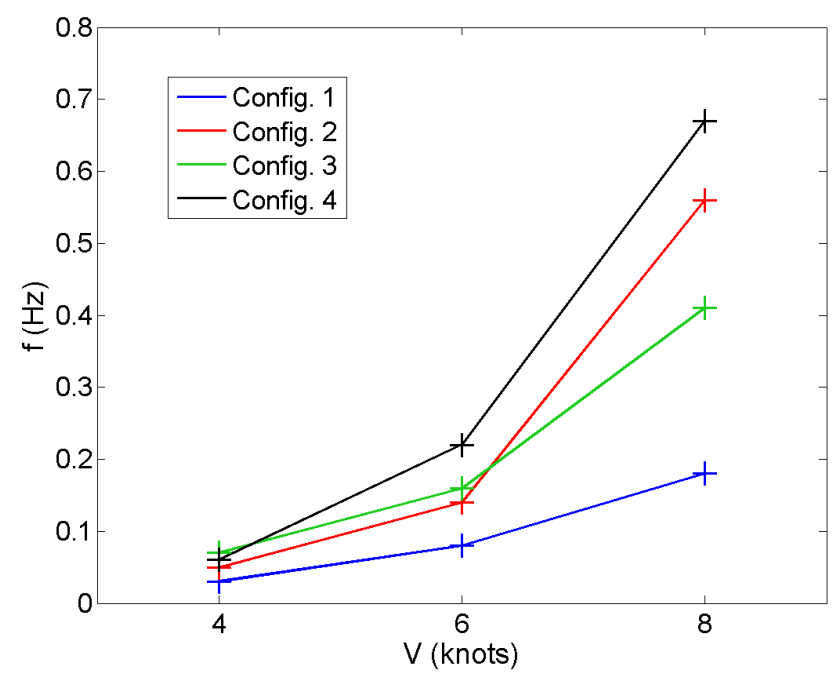

Figure 18: Influence of the velocity on the bubble cloud frequency.

The influence of the motions amplitude and frequency is shown on figure 19. The leftmost graph corresponds to the analysis of configurations 3 and 13 to 15 with respectively a motion amplitude coefficient of $0.75,1,1.25$ and 1.50. For each case the frequency of vortex shedding clouds is similar, close to $0.2 \mathrm{~Hz}$. Conversely the frequency of breaking wave clouds strongly increases with motion amplitude. The right hand side graph corresponds to the analysis of configurations 3 and 16 to 18 with respectively motion frequencies of $0.65,0.75,0.85$ and $1.00 \mathrm{~Hz}$. As for the effect of the wave frequency, the cloud frequency increases and seems to reach a maximum between 0.85 and $1.00 \mathrm{~Hz}$.

\subsection{Influence of the synchronism}

An important issue in the setup of base configuration 4 was to synchronize the model's motions with the waves. The phase was controlled by a variation of the delay between the start of the wavemaker and that of the hexapod. This delay corresponds to the time necessary for the first 

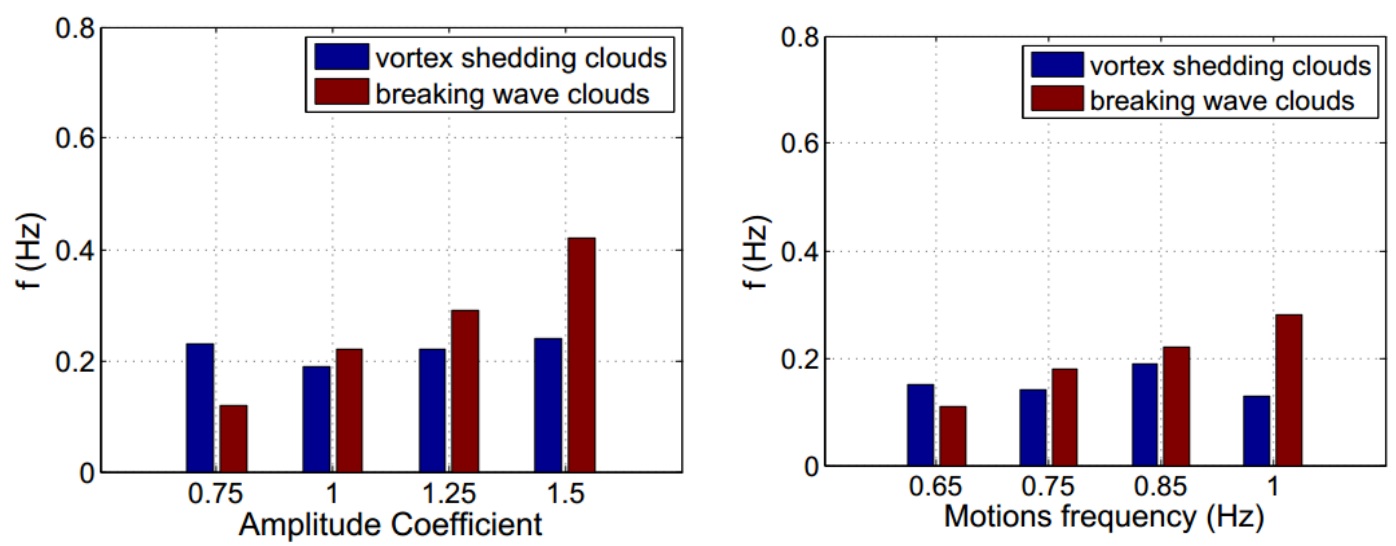

Figure 19: Influence of the motion parameters on the bubble cloud frequency. Left: motion amplitude coefficient. Right: motion frequency.

wave to travel from the wavemaker to the model. The numerical simulations showed that the heave and the wave at the center of gravity were in phase.

The influence of this parameter is shown in figure 20, corresponding to configurations 27 to 30 (with a mean phase shift, between the heave and the wave at the center of gravity, of $-90^{\circ},-5^{\circ}, 5^{\circ}$ and $30^{\circ}$, respectively). A phase shift of $5^{\circ}$ is not significant on the bubble clouds frequency. Nonetheless the two other cases demonstrate that this variable is a key parameter. For the phase shift of $-90^{\circ}$, the incoming waves simply follow the movements of the bow, and the impact is reduced or non-existent. In this case the bubble clouds frequency is reduced to $f=0.25 \mathrm{~Hz}$. Conversely for the phase shift of $30^{\circ}$, the bow exactly impacts the incoming wave, resulting in a breaking wave and bubble generation on almost every period $(f=0.84 \mathrm{~Hz}$ ). This phase shift is representative of real conditions: even if the ship's motions at sea directly respond to the most energetic waves, irregular waves may impact the bow and be a source of significant bubble clouds.

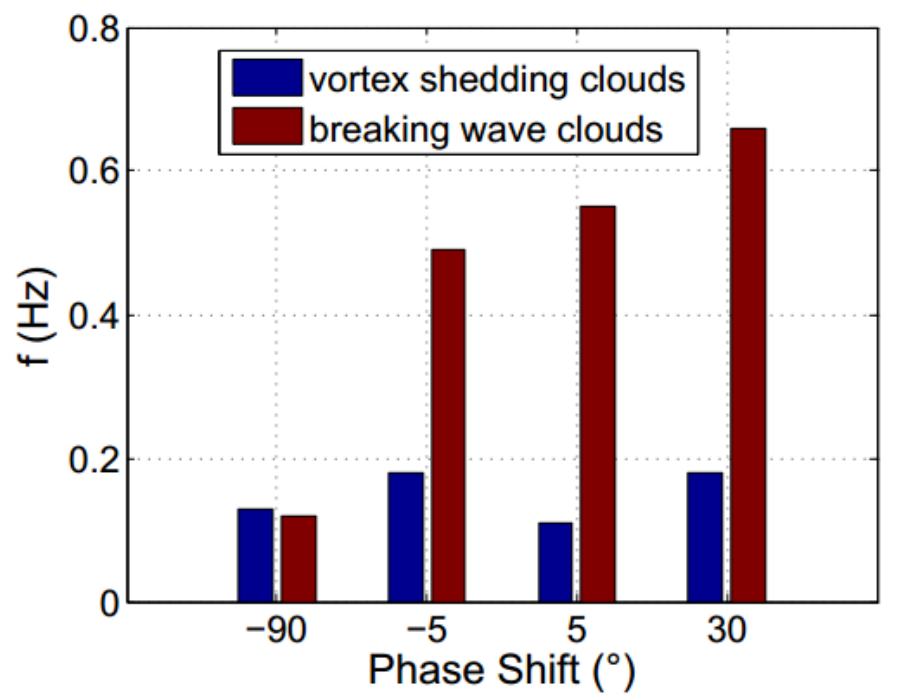

Figure 20: Influence of the phase shift on the bubble cloud frequency. 


\section{Discussions}

As mentioned throughout this article, the incoming turbulent flow generates a distortion of the flow in the vicinity of the bow, and the development of an air cavity leading to the air entrapment by vortex shedding. To the authors' knowledge, this phenomenon has not been described from classic towing tank tests. One of the main differences between these tests and ours is that the presence of the wavemaker in the circulating tank generates a very turbulent flow. The influence of this parameter is shown in table 2, which corresponds to test runs 1, 3, 31 and 32. In runs 31 and 32, the wavemaker is removed so the turbulent intensity of the flow (classically defined in Part I in Delacroix et al. (2016)) is $T I=3 \%$, closest to towing tank test classic set ups.

Table 2: Influence of the turbulence on the bubble cloud frequency.

\begin{tabular}{l|l|l}
\hline TI $(\%)$ & Config. 1 $(\mathrm{Hz})$ & Config. 2 $(\mathrm{Hz})$ \\
\hline 3 & 0 & 0.18 \\
\hline 15 & 0.06 & 0.40 \\
\hline
\end{tabular}

In the case with current only and $T I=3 \%$, there is no generation of bubbles. The phenomenon of air entrainment by vortex shedding disappears, which confirms the influence of the flow turbulence on this phenomenon. In the configuration with motions, the cloud frequency is also very low $(f=0.06 \mathrm{~Hz})$. On the opposite, when the turbulent intensity is closest to real ocean conditions $(T I=15 \%)$ the cloud frequencies are $0.18 \mathrm{~Hz}$ and $0.40 \mathrm{~Hz}$ for configurations 1 and 3 , respectively.

The fact that the wavemaker generates uncontrolled turbulence in the tank should be taken into account. The influence on bubble generation of a homogeneous isotropic turbulence could be different, even if the vortex shedding phenomenon is due to local variations in the flow.

The second item of discussion is related to similarity issues and results in the inability of direct extrapolation at real scale of the parameters' influence described above on the bubble cloud frequency. For instance, experiments from sea campaigns show that the influence of the ship's velocity on bubble sweep-down is not as significant as observed in section 4.2. It was observed in section 3.2 that bubbles never pass under the model's hull. This is mostly due to scale effects and the size of the bubbles in the tank, while the microscopic bubbles generated at sea may be dragged deeper. The size distribution of aerations detected by the image postprocessing method is given in figure 21 (left). In this graph the size distribution of bubbles (equivalent diameter) per $0.1 \mathrm{~mm}$ intervals is calculated by averaging all the images for which more than 10 bubbles are detected, from configuration 2 with current and waves. The number of detections is maximum for a $0.6 \mathrm{~mm}$ equivalent diameter, which is the detection minimum limit, and decreases dramatically to be very low above $2 \mathrm{~mm}$. There is no detection for an equivalent diameter greater than $4 \mathrm{~mm}$. These measurements are consistent with the rise velocity of bubble clouds observed. On the right-most, the final rise velocity of a single isolated and spherical bubble has been calculated according to the expression given by Comolet (1979), well fitted for bubble diameter $d<1 \mathrm{~mm}$ : 


$$
u_{\infty}=\frac{g \cdot \rho_{w}}{18 \cdot \mu_{w}} d_{e}^{2}
$$

where $g$ is the acceleration due to gravity, $d_{e}$ the equivalent bubble diameter, $\rho_{w}$ the density of water and $\mu_{w}$ the dynamic viscosity of water.
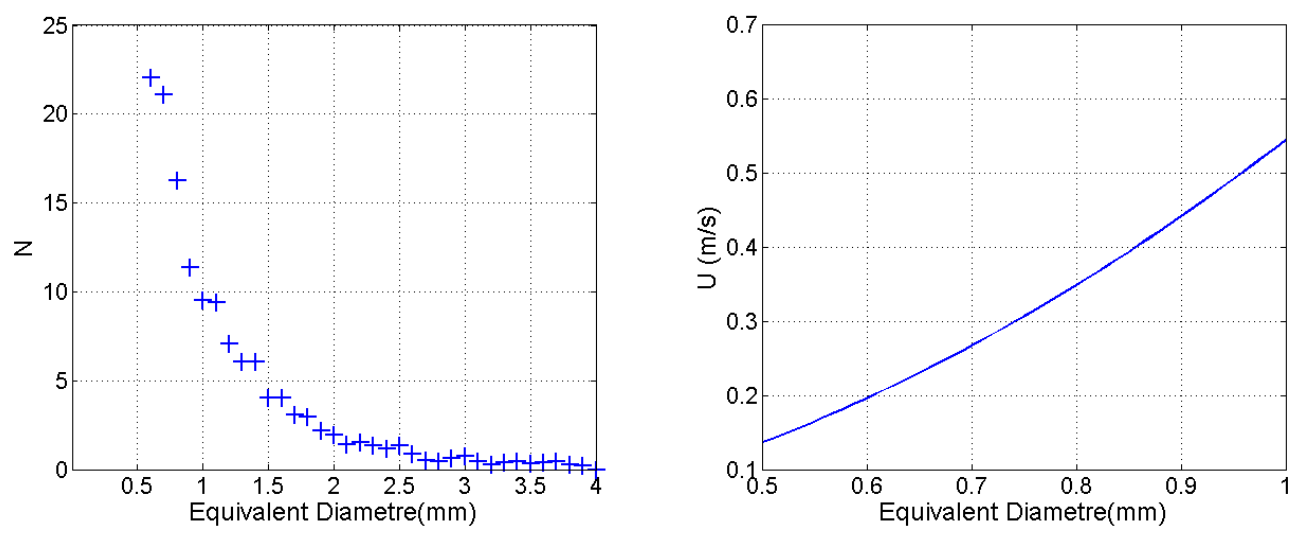

Figure 21: Left: Size distribution of the aerations detected for each image of the second configuration, with current and waves. Right: Theoretical bubble rise velocity.

The maximal rise velocity of a bubble cloud generated by breaking waves (section 3.2) is near $0.25 \mathrm{~m} / \mathrm{s}$, corresponding to the rise velocity of a bubble of diameter $d=0.67 \mathrm{~mm}$ close to the typical size of the observed bubbles.

Due to the Weber scale effects and because no correction was adopted for cross sectional influence, this bubble size distribution should be considered as indicative only. However, the mechanisms of air entrainment described in this article are similar to real sea events, even if the quantity of air entrained and the size distribution of bubbles (and consequently the rise velocity) are not.

\section{Conclusions and Perspectives}

The long term goal of this project is to develop a tool usable by research vessel designers, that would predict ship's behaviour in terms of bubble sweep-down. Through the two parts of this article, an experimental method developed in order to achieve this goal has been described. Several points have been highlighted:

- The wave and current flume tank is well-suited to reproduce the conditions of bubble sweep-down encountered at sea on a 1/30 model of the Pourquoi pas? ;

- The use of a hexapod to independently generate the model's motions allows to work on the model bow only, and facilitate the parametric study of the phenomenon ;

- The acquisition of high quality underwater images is facilitated in this configuration.

A method for image post-processing has been developed and used to characterize the bubble generation. Two phenomena have been described: air entrainment by vortex shedding or by 
breaking waves. A parametric study via a large number of experimental configurations has shown the influence of the main parameters:

- Vortex shedding clouds only exist in the tank with a high turbulent flow due to the wavemaker's presence upstream. The turbulent flow is favourable to bubble generation, and is the reason why bubble clouds are observed in the circulation tank but not in the towing tank for similar navigation conditions ;

- The waves are a determining factor on bubble generation and the clouds' frequency is directly proportional to the wave height;

- The considerable influence of the current's speed and the phase shift between waves and motions have also been demonstrated.

All these results have contributed to understanding the various physical mechanisms of bubble generation in a circulating tank with a model attached under a hexapod, and at sea despite the similarity issues discussed above. These tests have allowed the visualization and analysis of bubble generation around the bow of a model submitted to waves and motions. The database thus obtained is, to the authors' knowledge, unique and the properties calculated for the four configurations (area, depth and velocity of the clouds) could be used as a reference for the development of numerical models.

The other great advantage of these experiments is that the acquisition system also enable us to obtain a PIV database, leading to velocity fields (Druault, 2016). Simultaneous analysis of bubble generation and of the flow around the bow can thereby be conducted. These measurements could be used to characterize the turbulent structures, and the hydrodynamic events that lead to air entrainment mechanisms. These analyses should give more details on these mechanisms and should be used for the study and the prevention of bubble sweep down.

Acknowledgement The authors gratefully acknowledge the DGA (the French Government Agency for Defense) and Ifremer for the financial support of this co-financed $\mathrm{PhD}$ thesis.

We also would like to thank Thomas Bacchetti and Jean-Valery Facq for their assistance in the design and set-up of these experiments, and Alcino Ferreira for the proof-reading of the final paper.

\section{References}

Blenkinsopp C. E. and Chaplin J. R., 2007, Void fraction measurements in breaking waves, Proc. R. Soc. A, Vol.463, 3151-3170.

Bonmarin P., 1989, Geometric properties of deep-water breaking waves, Journal of Fluid Mechanics, Vol.209, 405-433.

Carrica P. M., Drew D., Bonetto F. and Lahey Jr R. T., 1999, A polydisperse model for bubbly two-phase flow around a surface ship, International Journal of Multiphase Flow, Vol.25, 257305. 
Chanson H. and Cummings P. D., 1994, Effects of Plunging Breakers on the Gas Contents in the Oceans, Marine Technology Society Journal, Vol.28, 22-32.

Cummings P. D. and Chanson H., 1997, Air entrainment in the developing flow region of plunging jet. Part 1: Theoretical development, Journal of Fluids Engineering, Vol.119, 597-602.

Cummings P. D. and Chanson H., 1997, Air entrainment in the developing flow region of plunging jet. Part 2: Experimental, Journal of Fluids Engineering, Vol.119, 603-608.

Cointe R. and Tulin M. P., 1994, A theory of steady breakers, Journal of Fluid Mechanics, Vol.276, 1-20.

Comolet R., 1979, Sur le mouvement d'une bulle de gaz dans un liquide (Gas bubble motion in a liquid medium), La Houille Blanche, Vol.1, 31-42 (in French)

Dabiri D. and Gharib M., 1997, Experimental investigation of the vorticity generation within a spilling water wave, Journal of Fluid Mechanics, Vol.330, 113-139.

Dantec Dynamic A/S, 2010, DynamicStudio User's Guide.

Deane G. B. and Stokes M. D., 2002, Scale dependence of bubble creation mechanisms in breaking waves, Nature, Vol.418, 839-844.

Delacroix S., 2015, Caractérisation de la génération et de la propagation de bulles autour de la carène de navires scientifiques, PhD Thesis, Université de Bretagne Occidentale.

Delacroix S., Germain G., Gaurier B., Billard JY., Experimental study of bubble sweep-down in wave and current circulationg tank, Part I Experimental set-up, under corrections in Ocean Engineering.

Delacroix S., Germain G., Berger L., Billard JY., 2016, Bubble sweep-down occurrence characterization on Research Vessels, Ocean Engineering, Vol. 111, 34-42.

Delhommeau G., Guilbaud M., David L., Yang C., Noblesse F., 2009, Boundary between unsteady and overturning ship bow wave regimes, Journal of Fluid Mechanics, Vol.620, 167-175.

Dussol D., Druault P., Mallat B., Delacroix S., Germain G., 2016, Automatic dynamic mask extraction for PIV images containing an unsteady interface, bubbles and a moving structure, Compte-Rendu Mécanique, In Press.

Dussol D., 2015, Etude de l'écoulement autour de l'étrave des navires océanogrphiques (in French), Master's thesis, Université Pierre et Marie Curie-Paris 6.

Duncan J. H., 1981, An Experimental Investigation of Breaking Waves Produced by a Towed Hydrofoil, Proc. R. Soc. A, Vol.377, 331-348.

Duncan J. H., 1983, The breaking and non-breaking wave resistance of a two-dimensional hydrofoil, Journal of Fluid Mechanics, Vol.126, 507-520. 
Kiger K. T. and Duncan J. H., 2012, Air-entrainment mechanisms in plunging jets and breaking waves, Annu. Rev. Fluid Mech., Vol.44, 563-596.

Lamarre E. and Melville W. K., 1994, Void-fraction measurements and sound-speed fields in bubble plumes generated by breaking waves, J. Acoust. Soc. Am., Vol.95, 1317-1328.

Lin J. C. and Rockwell D., 1995, Evolution of a quasi-steady breaking wave, Journal of Fluid Mechanics, Vol.302, 29-44.

Loewen M. R., O’Dor M. A. and Skafel M. G., 1996, Bubbles entrained by mechanically generated breaking waves, Journal of Geophysical Research, Vol.101, 759-769.

Ma J., Oberai A. A., Hyman M. C., Drew D. A., Lahey Jr R. T., 2011, Two-fluid modeling of bubbly flows around surface ships using a phenomenological subgrid air entrainment model, Computers \& Fluids, Vol.52, 50-57.

Moraga F. J., Carrica P. M., Drew D. A., Lahey Jr R. T., 2008, Air entrainment model for breaking bow waves and naval surface ships, Computers \& Fluids, Vol.37, 281-298.

Noblesse F., Delhommeau G., Guilbaud M., Hendrix D., Yang C., 2008, Simple analytical relations for ship bow waves, Journal of Fluid Mechanics, Vol.600, 105-132.

Noblesse F., Delhommeau G., Guilbaud M., Liu H., Wan D., Yang C., 2013, Ship bow waves, Journal of Hydrodynamics, Vol.25, 491-501.

Prewitt J., 1970, Object enhancement and extraction, Academic Press.

Shakeri M., Tavakolinejad M. and Duncan J. H., 2009, An experimental investigation of divergent bow waves simulated by a two-dimensional plus temporal wave marker technique, Journal of Fluid Mechanics, Vol.634, 217-243.

Tavakolinejad M., 2010, Air bubble entrainment by breaking bow waves simulated by a $2 \mathrm{D}+\mathrm{T}$ technique, PhD Thesis, University of Maryland.

Waniewski T. A., Brennen C. E. and Raichlen F., Measurements of Air Entrainment by Bow Waves, Journal of Fluids Engineering, Vol.123, 57-63. 